\title{
THE SYMPLECTIC FLOER HOMOLOGY OF A DEHN TWIST
}

\author{
PAul SeIDel
}

Abstract. We compute examples of symplectic Floer homology in the lowest dimension i.e. for surfaces.

\section{Statement}

In two dimensions a diffeomorphism is symplectic if and only if it preserves volumes. As a consequence, the symplectic geometry of surfaces lacks many of the interesting phenomena which are encountered in higher dimensions. For example, two symplectic automorphisms of a closed surface are symplectically isotopic iff they are homotopic, by a theorem of Moser [4]. On the other hand symplectic fixed point theory is nontrivial even in dimension 2, as shown by the Poincaré-Birkhoff theorem [1]. So while symplectic Floer homology on surfaces is essentially a topological invariant, it is not clear how much information it contains. In this note we compute it in some simple cases.

Let $\Sigma$ be an oriented closed connected surface of genus $\geq 2$ and $C \subset$ $\Sigma$ a closed 1-submanifold whose components $C_{1}, \ldots C_{n}$ are labeled with $\sigma_{1}, \ldots \sigma_{n} \in\{ \pm 1\}$. We assume that no component of $\Sigma-C$ is a disc, and that both boundary circles of any component which is an annulus carry the same sign. Let $T_{i}$ be the positive Dehn twist along $C_{i}$. This is an automorphism of $\Sigma$ defined as follows: choose an oriented tubular neighbourhood

$$
c_{i}:[-2 ; 2] \times S^{1} \longrightarrow \Sigma,
$$

such that $c_{i}\left(0 \times S^{1}\right)=C_{i}$ and a monotone function $\psi \in C^{\infty}(\mathbb{R}, \mathbb{R})$ such that $\psi(t)=0$ for $t \leq-1$ and $\psi(t)=1$ for $t \geq 1$. Then

$$
c_{i}^{-1} T_{i}\left(c_{i}(s, t)\right)=(s, t-\psi(s)),
$$

which we extend trivially over $\Sigma$. Actually we will choose the $c_{i}$ to have disjoint images; then the standard volume forms given by $c_{i}$ can be extended to an $\omega \in \Omega^{2}(\Sigma)$ which is fixed by all $T_{i}$, and

$$
T=T_{1}^{\sigma_{1}} T_{2}^{\sigma_{2}} \ldots T_{n}^{\sigma_{n}}
$$

Received March 19, 1996.

Supported by the German Academic Exchange Service. 
is a symplectic automorphism of $(\Sigma, \omega)$. As we will see below $T$ has welldefined symplectic Floer cohomology groups ${ }^{1} H F^{*}(T)$. Moreover there is a 'quantum' product

$$
H^{*}(\Sigma) \otimes H F^{*}(T) \longrightarrow H F^{*}(T) .
$$

Our result is

Theorem 1. Let $C_{+}$be the union of all $C_{i}$ such that $\sigma_{i}=+1, C_{-}=$ $C-C_{+}$. Then there is an isomorphism of $H^{*}(\Sigma)$-modules

$$
H F^{*}(T) \cong H^{*}\left(\Sigma-C_{-}, C_{+}\right),
$$

where $H^{*}(\Sigma)$ acts on the r.h.s. by cup product.

Actually there is some ambiguity in the grading of the Floer homology groups; $H F^{*}(T)$ can be split into several parts, and the grading on each of these parts is only determined up to an even constant. The theorem says that for a suitable choice of these constants, the isomorphism preserves the grading.

Example 2. Take $C$ to be a single curve which divides $\Sigma$ into two parts, labeled with +1 . Then

$$
H F^{2}(T)=\mathbb{Z}^{2}, H F^{1}(T)=\mathbb{Z}^{2 g}, H F^{0}(T)=0,
$$

where $g=\operatorname{genus}(\Sigma)$. It is interesting to compare this with the instanton Floer homology of the mapping torus $T_{T} \Sigma$. For $g=2$ Callahan (unpublished) showed that $H F_{\text {inst }}^{*}\left(T_{T} \Sigma\right)$ and $H F_{\text {inst }}^{*}\left(\Sigma \times S^{1}\right)$ are isomorphic as $\mathbb{Z} / 4$-graded abelian groups, but are distinguished by their multiplicative structures. The same thing is true in our case if we reduce the grading $\bmod 2$ :

$$
H F^{e v}(T) \cong H F^{e v}\left(\operatorname{id}_{\Sigma}\right), H F^{o d d}(T) \cong H F^{o d d}\left(\operatorname{id}_{\Sigma}\right),
$$

but $H F^{2}(\Sigma)$ acts trivially on $H F^{*}(T)$ and nontrivially on $H F^{*}\left(\operatorname{id}_{\Sigma}\right)$. Similarly $H F^{*}(T)$ is not isomorphic to $H F^{*}\left(T^{-1}\right)$ as $\mathbb{Z} / 2$-graded $H^{*}(\Sigma)$ module because $H^{1}(\Sigma)$ acts trivially on $H F^{e v}(T)$ and nontrivially on $H F^{o d d}(T)$.

In fact one can see symplectic Floer homology on surfaces as a simple model for the instanton theory; this point of view will be pursued further in subsequent work.

We will now discuss briefly the questions that arise when trying to set up Floer homology for an arbitrary symplectic automorphism $f$ of $\Sigma$. Floer

\footnotetext{
${ }^{1}$ Note that our convention differs from that of [2] by a sign: our $H F^{*}(T)$ is their $H F^{*}\left(T^{-1}\right)$. This change was made in order to make the notation coherent with that for time-dependent Hamiltonians [3].
} 
homology is an analogue of Morse theory for a certain closed one-form on the space

$$
\Lambda_{f} \Sigma=\left\{\alpha \in C^{\infty}(\mathbb{R}, M) \mid \alpha(s)=f(\alpha(s+1))\right\} .
$$

The one-form is

$$
d a_{\alpha}(\xi)=\int_{0}^{1} \omega\left(\frac{d \alpha}{d s}, \xi(s)\right) d s .
$$

One problem is familiar from ordinary Morse theory: $d a$ might have periods. A loop in $\Lambda_{f} \Sigma$ is a map

$$
h: S^{1} \times[0 ; 1] \longrightarrow \Sigma,
$$

such that $h(s, 0)=f(h(s, 1))$, and

$$
\langle d a,[h]\rangle=-\int_{S^{1} \times[0 ; 1]} h^{*} \omega .
$$

To prove that these periods vanish for $f=T$, we need the first part of the following elementary

Lemma 3. Let $T$ be a product of Dehn twists as above.

(i) Denote by $\Lambda \Sigma$ the free loop space. If $L \in \pi_{0}(\Lambda \Sigma)$ is fixed by $T_{*}$ there is an $l \in L$ such that $\operatorname{im}(l) \subset \operatorname{Fix}(T)$.

(ii) Let $x, y$ be fixed points of $T$, and $\Omega_{x, y} \Sigma$ the space of paths from $x$ to $y$. If $A \in \pi_{0}\left(\Omega_{x, y} \Sigma\right)$ is fixed by $T_{*}$ there is an $a \in A$ such that $\operatorname{im}(a) \subset \operatorname{Fix}(T)$.

Given this, we can represent any element of $\pi_{1}\left(\Lambda_{T} \Sigma\right)$ by an $h$ such that $h(s, 0)=h(s, 1)$. Since genus $(\Sigma) \geq 2$ this implies $\int h^{*} \omega=0$.

A second point (which does not arise in finite dimensions) is that the relative index of two critical points may depend on a path connecting them, which makes it impossible to find a $\mathbb{Z}$-grading for the Floer complex. The problem is caused by a class $\mu \in H^{1}\left(\Lambda_{f} \Sigma, \mathbb{Z}\right)$ which is defined as follows: let $T_{f} \Sigma$ be the mapping torus of $f$. There is a map

$$
d: H^{2}\left(T_{f} \Sigma\right) \longrightarrow H^{1}\left(\Lambda_{f} \Sigma\right),
$$

and $\mu=d\left(c_{1}\left(T_{f} \Sigma\right)\right)$. More concretely any loop in $\Lambda_{f} \Sigma$ gives a map

$$
h: S^{1} \times S^{1} \longrightarrow T_{f} \Sigma,
$$

as above and the value of $\mu$ on it is $\int_{S^{1} \times S^{1}} h^{*} c_{1}\left(T_{f} \Sigma\right)$. Now we can use the same argument as before to show that $\mu$ vanishes in our examples.

Finally the main difficulty in Floer homology for general symplectic manifolds is the noncompactness of moduli spaces due to bubbling off of holomorphic spheres, which does not occur in our case since $\pi_{2}(\Sigma)=0$. 


\section{Proof}

The proof of Theorem 1 uses the familiar technique of 'stretching the neck' (note however that it is here applied to the target manifold $\Sigma$ ). First let

$$
\Sigma^{\prime}=\Sigma-\bigcup_{i=1}^{n} c_{i}(]-1 ;+1\left[\times S^{1}\right)
$$

and denote by $\omega^{\prime}$ the restriction of $\omega$ to $\Sigma^{\prime}$. Choose an $\omega^{\prime}$-compatible complex structure $J$ on $\Sigma^{\prime}$ which agrees with the standard one given by the $c_{i}$ near $\partial \Sigma^{\prime}$. Finally we need a Morse function $h: \Sigma^{\prime} \longrightarrow \mathbb{R}$ satisfying $h\left(c_{i}( \pm 1, t)\right)=0$ for all $t$ and

$$
\left(c_{i}^{*} d h\right)(s, t)=\left\{\begin{array}{ll}
-\sigma_{i} \epsilon d s & -2 \leq s \leq-1 \\
\sigma_{i} \epsilon d s & 1 \leq s \leq 2
\end{array},\right.
$$

for some small $\epsilon>0$. This defines a Hamiltonian flow $\phi_{t}$ on $\Sigma^{\prime}$ and complex structures $J_{t}=\phi_{t}^{*} J$ which are equal to $J$ near the boundary.

Now consider the surfaces $\left(\Sigma^{R}, \omega^{R}\right.$ ) (for $R \geq 2$ ) obtained by replacing the necks $\operatorname{im}\left(c_{i}\right) \subset \Sigma$ by $N_{i}^{R}=[-R ; R] \times S^{1}$ with the standard volume form. $\Sigma^{\prime} \subset \Sigma^{R}$ in an obvious way, and there are unique complex structures $J_{t}^{R}$ which are standard on the $N_{i}^{R}$ and agree with $J_{t}$ on $\Sigma^{\prime}$. Similarly let $h^{R}: \Sigma^{R} \longrightarrow \mathbb{R}$ be the function whose differential on $N_{i}^{R}$ is

$$
d h^{R}(s, t)=\sigma_{i} \epsilon(2 \psi(s)-1) d s,
$$

and which agrees with $h$ on $\Sigma^{\prime}$ (such a function exists for suitable choices of $\psi$ ), and $\phi_{t}^{R}$ its Hamiltonian flow.

We define symplectic diffeomorphisms $T^{R}$ of $\Sigma^{R}$ such that on $N_{i}^{R}$

$$
T^{R}(s, t)=\left(s, t-\sigma_{i} \psi(s)\right),
$$

extending it by the identity as before, and perturbations $T_{0}^{R}=\phi_{1}^{R} T^{R}$. This means that on $N_{i}^{R}$

$$
T_{0}^{R}(s, t)=\left(s, t+\sigma_{i}((2 \epsilon-1) \psi(s)-\epsilon)\right),
$$

has no fixed points.

$T^{R}$ can be obtained from $T$ by a deformation of the symplectic form; this implies that

$$
H F^{*}(T) \cong H F^{*}\left(T_{0}^{R}\right),
$$

for all $R$.

Recall that the Floer cohomology of $T_{0}^{R}$ is constructed using the moduli spaces $\mathcal{M}^{R}\left(x_{-}, x_{+}\right)$of maps

$$
u: \mathbb{R} \times \mathbb{R} \longrightarrow \Sigma^{R},
$$


such that

$$
\begin{aligned}
& u(s, t)=T_{0}^{R}(u(s, t+1)), \\
& \frac{d u}{d s}+J_{t}^{R}(u(s, t)) \frac{d u}{d t}=0, \\
& \lim _{s \longrightarrow \pm \infty} u(s, t)=x_{ \pm},
\end{aligned}
$$

where $x_{-}, x_{+} \in \operatorname{Fix}\left(T_{0}^{R}\right)$. The main step in the proof is then the following

Lemma 4. Let $R$ be large. Then for any $x_{-}, x_{+} \in \operatorname{Fix}\left(T_{0}^{R}\right)$ and $u \in$ $\mathcal{M}^{R}\left(x_{-}, x_{+}\right)$we have

$$
u(\mathbb{R} \times \mathbb{R}) \subset \operatorname{Fix}\left(T^{R}\right) .
$$

This means that $u$ never reaches the regions inside $[-1 ; 1] \times S^{1} \subset N_{i}^{R} \subset$ $\Sigma^{R}$ where the Dehn twist actually takes place.

To prove this consider first points $x_{+}, x_{-} \in \operatorname{Fix}\left(T_{0}^{R}\right)$ which lie in different components of $\Sigma^{\prime}$. It follows from Lemma 3 (ii) that the constant paths at $x_{-}, x_{+}$lie in different components of $\Lambda_{T_{0}^{R}} \Sigma^{R}$. Therefore $\mathcal{M}^{R}\left(x_{-}, x_{+}\right)=\emptyset$ (this separation mechanism is well-known from Nielsen fixed point theory).

Now assume that $x_{ \pm}$lie in the same component of $\Sigma^{\prime}$, and that there are $R_{i} \longrightarrow \infty$ and $u^{i} \in \mathcal{M}^{R_{i}}\left(x_{-}, x_{+}\right)$such that $u^{i}(\mathbb{R} \times \mathbb{R}) \not \subset \operatorname{Fix}\left(T^{R_{i}}\right)$. Their energy is

$$
E\left(u^{i}\right)=\int_{\mathbb{R} \times[0 ; 1]}\left|d u^{i}\right|^{2}=a\left(x_{-}\right)-a\left(x_{+}\right)=h\left(x_{-}\right)-h\left(x_{+}\right),
$$

and therefore uniformly bounded (here we used the fact that $d a$ has an integral $a$ ). It follows easily that $\left|d u^{i}\right|_{\infty}$ has to be unbounded. Let $z_{i} \in$ $\mathbb{R} \times[0 ; 1]$ be a point where $\left|d u^{i}\right|$ is maximal. Then for a suitable subsequence

$$
C_{i}=\left|d u^{i}\left(z_{i}\right)\right| \longrightarrow \infty \quad \text { as } i \longrightarrow \infty,
$$

and one of the following holds:

Case 1: the distance of $u^{i}\left(z_{i}\right)$ to $\Sigma^{\prime} \subset \Sigma^{R_{i}}$ goes to $\infty$ as $i \longrightarrow \infty$. Then the rescaled maps

$$
\tilde{u}^{i}(z)=u^{i}\left(C_{i}^{-1} z+z_{i}\right),
$$

converge on compact subsets to a nonconstant holomorphic curve $\tilde{u}: \mathbb{C} \longrightarrow$ $\mathbb{R} \times S^{1}$. But such a curve cannot have finite energy.

Case 2: $u^{i}\left(z_{i}\right)$ stays within a finite distance of $\Sigma^{\prime}$. Then by rescaling we would obtain a nonconstant $J_{t}$-holomorphic sphere (for some $t$ ) in the surface obtained from $\Sigma^{\prime}$ by attaching a semi-infinite cylinder to each boundary component, which is again impossible. 
Theorem 1 now follows by a standard argument [6, Theorem 7.3]: for large $R$ and small $\epsilon, \mathcal{M}^{R}\left(x_{+}, x_{-}\right)$is homeomorphic to the space of $u$ : $\mathbb{R} \longrightarrow \Sigma^{\prime}$ such that

$$
\frac{d u}{d t}=-\nabla_{J} h(u), \lim _{t \longrightarrow \pm \infty} u(t)=x_{ \pm},
$$

and for a generic $J$ every solution is regular. This gives an isomorphism between the Floer chain complex of $T_{0}^{R}$ and the Morse-theoretic chain complex associated to $h$ on $\Sigma^{\prime}$, whose cohomology is $H^{*}\left(\Sigma-C_{-}, C_{+}\right)$.Using the 'extrinsic' definition of the quantum product [5] it is easy to see that this is an isomorphism of $H^{*}(\Sigma)$-modules.

\section{References}

1. G. D. Birkhoff, Proof of Poincaré's geometric theorem, Collected mathematical papers vol. 1, Amer. Math. Soc., 1950, pp. 673-681.

2. S. Dostoglou and D. Salamon, Self dual instantons and holomorphic curves, Annals of Math. 139 (1994), 581-640.

3. A. Floer, Symplectic fixed points and holomorphic spheres, Comm. Math. Phys. 120 (1989), 575-611.

4. J. Moser, On the volume elements on a manifold, Trans. Amer. Math. Soc. 120 (1965), 286-294.

5. Y. Ruan and G. Tian, Bott-type symplectic Floer cohomology and its multiplication structures, Math. Res. Lett. 2 (1995), 203-219.

6. D. Salamon and E. Zehnder, Morse theory for periodic solutions of Hamiltonian systems and the Maslov index, Comm. Math. Phys. 45 (1992), 1303-1360.

Mathematical Institute, 24-29 St Giles', Oxford OX1 3LB, ENGLAND

E-mail address: seidel@maths.ox.ac.uk 